\title{
Perfusion MRI-Based Fractional Tumor Burden Differentiates between Tumor and Treatment Effect in Recurrent Glioblastomas and Informs Clinical Decision-Making
}

\author{
(D) M. Iv, (D) X. Liu, (D). Lavezo, (D)A.J. Gentles, (D) R. Ghanem, (D). Lummus, (DD.E. Born, (D) S.G. Soltys, (D). Nagpal, (D) R. Thomas, \\ (D). Recht, and DN. Fischbein
}

\begin{abstract}
BACKGROUND AND PURPOSE: Fractional tumor burden better correlates with histologic tumor volume fraction in treated glioblastoma than other perfusion metrics such as relative CBV. We defined fractional tumor burden classes with low and high blood volume to distinguish tumor from treatment effect and to determine whether fractional tumor burden can inform treatment-related decision-making.
\end{abstract}

MATERIALS AND METHODS: Forty-seven patients with high-grade gliomas (primarily glioblastoma) with recurrent contrast-enhancing lesions on DSC-MR imaging were retrospectively evaluated after surgical sampling. Histopathologic examination defined treatment effect versus tumor. Normalized relative CBV thresholds of 1.0 and 1.75 were used to define low, intermediate, and high fractional tumor burden classes in each histopathologically defined group. Performance was assessed with an area under the receiver operating characteristic curve. Consensus agreement among physician raters reporting hypothetic changes in treatmentrelated decisions based on fractional tumor burden was compared with actual real-time treatment decisions.

RESULTS: Mean low fractional tumor burden, high fractional tumor burden, and relative CBV of the contrast-enhancing volume were significantly different between treatment effect and tumor $(P=.002, P<.001$, and $P<.001)$, with tumor having significantly higher fractional tumor burden and relative CBV and lower fractional tumor burden. No significance was found with intermediate fractional tumor burden. Performance of the area under the receiver operating characteristic curve was the following: high fractional tumor burden, 0.85; low fractional tumor burden, 0.7; and relative CBV, 0.81. In comparing treatment decisions, there were disagreements in $7 \%$ of tumor and $44 \%$ of treatment effect cases; in the latter, all disagreements were in cases with scattered atypical cells.

CONCLUSIONS: High fractional tumor burden and low fractional tumor burden define fractions of the contrast-enhancing lesion volume with high and low blood volume, respectively, and can differentiate treatment effect from tumor in recurrent glioblastomas. Fractional tumor burden maps can also help to inform clinical decision-making.

ABBREVIATIONS: FTB = fractional tumor burden; $\mathrm{HGG}=$ high-grade glioma; ICC = intraclass correlation coefficient; rCBV = relative cerebral blood volume; $\mathrm{TE}=$ treatment effect

$\Delta$

$\mathrm{n}$ important challenge in the care of patients with high-grade gliomas (HGGs) following conventional therapy with

\section{Received May 9, 2019; accepted after revision August 1.}

From the Departments of Neuroimaging and Neurointervention (M.I., N.F.), Pathology (J.L., R.G., S.L., D.E.B.), Medicine (Biomedical Informatics Research) (A.J.G.), Radiation Oncology (S.G.S.), and Neurology (Neuro-Oncology) (S.N., R.T., L.R.) Stanford University, Stanford, California; and Department of Neurosurgery (X.L.), Shengjing Hospital of China Medical University, Shenyang, China.

Michael Iv and Xiaobai Liu are co-first authors and contributed equally to this work.

Please address correspondence to Michael Iv, MD, Stanford University Medical Center, Department of Radiology, 300 Pasteur Dr, Grant Bldg, Room S031E, Stanford, CA 94305; e-mail: miv@stanford.edu; @Michael_Iv_MD

Indicates article with supplemental on-line photos.

http://dx.doi.org/10.3174/ajnr.A6211 maximal safe surgical resection and chemoradiation is the differentiation of tumor and treatment effect (TE). The current practice standard, Response Assessment in Neuro-Oncology criteria, to determine the response to therapy of a tumor is largely based on the assessment of T2/FLAIR signal extent and the size of T1 gadolinium enhancement on MR imaging across time. ${ }^{1}$ An increase in T2/FLAIR signal and contrast enhancement following treatment does not, however, always indicate tumor progression. Thus, perfusion imaging markers such as relative cerebral blood volume (rCBV) have been thoroughly investigated and used to differentiate tumor from TE. ${ }^{2-4}$ More recent studies have demonstrated that another perfusion-derived metric, fractional tumor burden (FTB), which is defined as the volume fraction of tumor voxels above a specified rCBV threshold, has similar potential. ${ }^{2,3,5}$ 
Despite their promise, MR imaging perfusion-derived metrics have yet to be widely adopted. Reasons for this include interoperator subjectivity in producing rCBV values (often based on "hot spot" ROI analysis), lack of clinically validated parameters to differentiate tumor from TE, and lack of standardization of imaging-acquisition techniques and postprocessing software across different sites. ${ }^{6}$ Specifically, the hot spot ROI methodology is largely based on manually placing an ROI in an area of tumor with the highest rCBV on a single image. ${ }^{7-9}$ This method, however, underrepresents the entire volume and heterogeneity of the tumor. Assessment of whole-tumor perfusion, for example, with FTB mitigates this issue by providing per-voxel measurements rather than computing 1 value to represent the entire contrastenhancing lesion. Even with this technique, however, an rCBV threshold defining tumor versus TE is needed. A previous study evaluating stereotactic biopsy specimens of recurrent glioblastoma demonstrated that $\mathrm{rCBV}$ of $\leq 1.0$ distinguished tumor from TE with $100 \%$ accuracy. ${ }^{2}$ Another study found similar findings, with a threshold of 1.13 yielding $82 \%$ sensitivity and $90 \%$ specificity. ${ }^{3}$

Apart from using rCBV to differentiate TE from tumor, higher rCBV thresholds have been used to identify more aggressive tumors. One of the earliest glioma studies using a single rCBV threshold found that tumors with an $\mathrm{rCBV}$ of $>1.75$ portended a worse prognosis. ${ }^{8}$ Another study found that rCBV of $\geq 1.8$ (despite rCBV being estimated by the negative enhancement integral on T2WI) best distinguished tumor burden when it was $>20 \%$ of the entire tumor. ${ }^{10}$ Discrepancies in previously reported rCBV thresholds, ${ }^{2,3,8,10}$ therefore, are not always due to lack of standardized methodologies but may be related to the specific question being asked.

In this study, we evaluated the utility of quantitative FTB of the entire contrast-enhancing lesion volume in patients with suspected recurrent HGGs. We hypothesize that the use of $2 \mathrm{rCBV}$ values (1.0, which has been found to effectively differentiate TE from tumor, and 1.75, which has been shown to indicate aggressive tumor) to define low and high fractional tumor burden, respectively, would be effective in distinguishing recurrent tumor from TE. We also assessed whether qualitative interpretation of FTB among 5 physicians agrees with the histopathologic diagnosis and whether FTB can be used to inform treatment-related decision-making.

\section{MATERIALS AND METHODS \\ Patients}

This retrospective study was approved by Stanford University's institutional review board. We evaluated adults with suspected HGG recurrence after previous surgical resection followed by standard chemoradiation between January 2007 to June 2018. Inclusion criteria were the following: 18 years of age or older with HGG initially treated with conventional therapy, enlarging or new contrast-enhancing mass on follow-up DSC-MR imaging, surgical resection or biopsy of the mass, and availability of tissue specimens for histopathologic examination. All patients who had a resection had $>90 \%$ or gross total resection as determined qualitatively by the amount of residual contrast enhancement on immediate postsurgical T1WI. Exclusion criteria included non- contrast-enhancing tumor on MR imaging, marked susceptibility related to blood or surgical material on raw precontrast DSC images, low-grade gliomas, and any oligodendroglioma. Oligodendrogliomas were excluded because elevated intratumoral $\mathrm{rCBV}$ has been shown to relate to fine capillaries and is not necessarily indicative of aggressive tumor. ${ }^{11}$ Patients on bevacizumab at the time of an operation for suspected recurrence were not excluded because the presence of an enlarging contrast-enhancing mass while on bevacizumab suggests a refractory response to antiangiogenic therapy. ${ }^{12}$ After screening and assessment of eligibility, 47 patients were included (Online Fig 1). Clinical demographics, histopathologic and molecular information, and treatment history were obtained through the electronic medical record.

\section{Perfusion MR Imaging Acquisition}

MRIs were performed on a $1.5 \mathrm{~T}(n=28$, Signa Explorer; GE Healthcare, Milwaukee, Wisconsin) or 3T $(n=19$, Discovery MR750; GE Healthcare) scanner. Images were acquired as part of the brain tumor protocol of our institution, which varied across the years. However, all examinations included pre- and postgadolinium axial 2D-T1-weighted spin-echo or 3D-T1-weighted inversion recovery echo-spoiled gradient-echo BRAin VOlume (BRAVO) images (GE Healthcare). DSC imaging was performed in each patient, and during the study period, it was acquired with nonpreload single-echo gradient EPI (parameters: TR/TE $=1800$ / $35-40 \mathrm{~ms}$, section thickness $=5 \mathrm{~mm}, 0$ skip with 20 images covering the brain, flip angle $=60^{\circ}$, matrix $=96 \times 128 \mathrm{~mm}$, FOV $=$ 220-240 mm). A dynamic bolus was acquired using a full dose of a gadolinium $(0.1 \mathrm{mmol} / \mathrm{kg})$, which was administered intravenously by a power injector at a rate of $4-5 \mathrm{~mL} / \mathrm{s}$.

\section{Image Processing and Quantitative Analysis}

We used a workstation equipped with OsiriX MD (Version 7.0; http://www.osirix-viewer.com) and a commercially available plug-in (IB Neuro, Version 2.0; Imaging Biometrics, Elm Grove, Wisconsin), which uses well-established and previously published methods, including a leakage-correction algorithm, to process perfusion data and calculate rCBV and FTB. ${ }^{2,3,5,13-15}$ For semiautomated image analysis, we used IB Rad Tech (Version 2.0; Imaging Biometrics), which is a workflow engine that generates quantitative $\Delta \mathrm{T} 1$ and FTB maps from the IB Delta Suite (Version 2.0; Imaging Biometrics), and IB Neuro plug-ins. The overall workflow, which has been described previously, ${ }^{2}$ is highlighted as follows: 1) The volume of contrast enhancement was determined from $\Delta \mathrm{T} 1$ maps, which are standardized difference maps computed from the difference of coregistered pre- and postcontrast T1-weighted images; ${ }^{14-17}$ 2) postcontrast T1 images were coregistered to the raw DSC images; 3 ) the contrast-enhancing VOI was transferred to the rCBV map; 4) normalization was performed by drawing and taking the average of two $5 \times 5 \mathrm{~mm}^{2}$ ROIs in the contralateral normal appearing white matter; and 5) output rCBV and FTB maps, in which lesion mask voxels were used to classify areas of contrast enhancement on the basis of predefined rCBV thresholds, were subsequently generated. As previously stated, we selected thresholds of 1.0 and 1.75 to define 3 FTB classes: $\mathrm{FTB}_{\text {low }}$, percentage of contrast-enhancing voxels with 

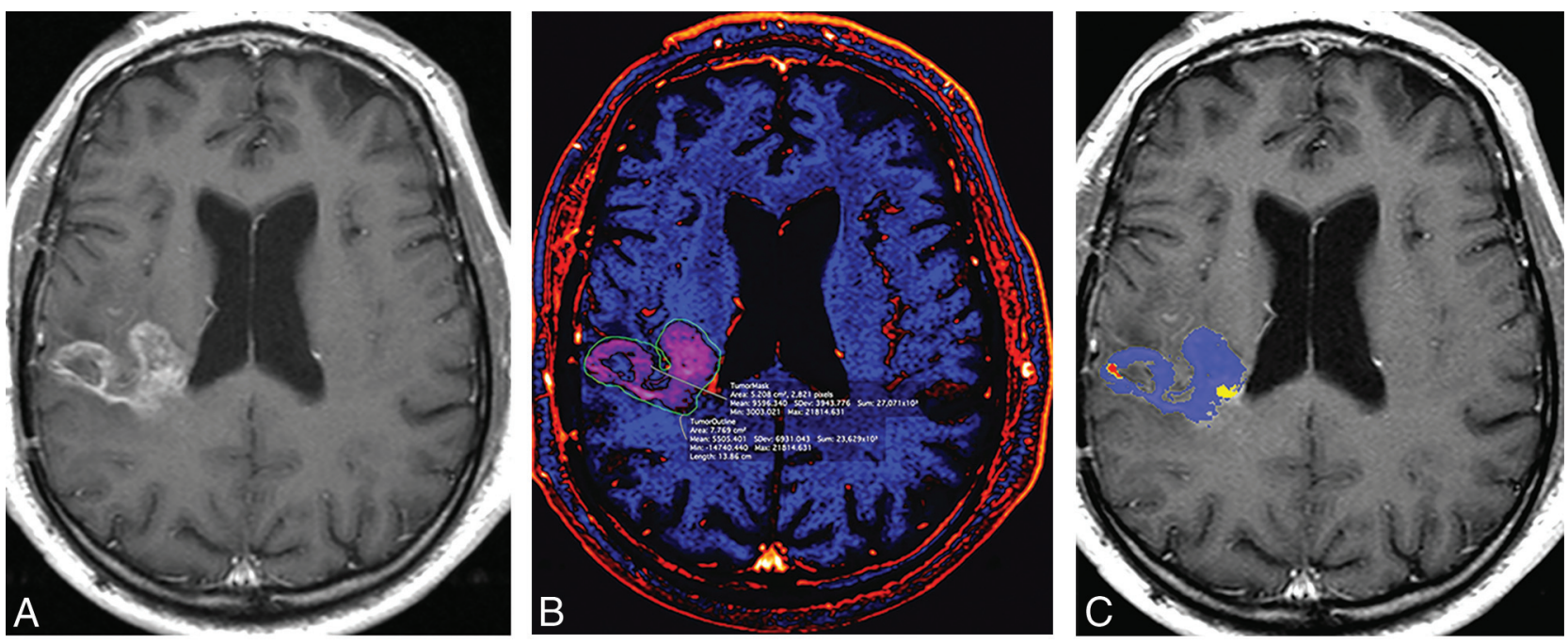

Total $N=6425$ Mean= 0.405768 StdDe $v=0.377849$ Median $=0.275749$ Skeuness $=2.56928$

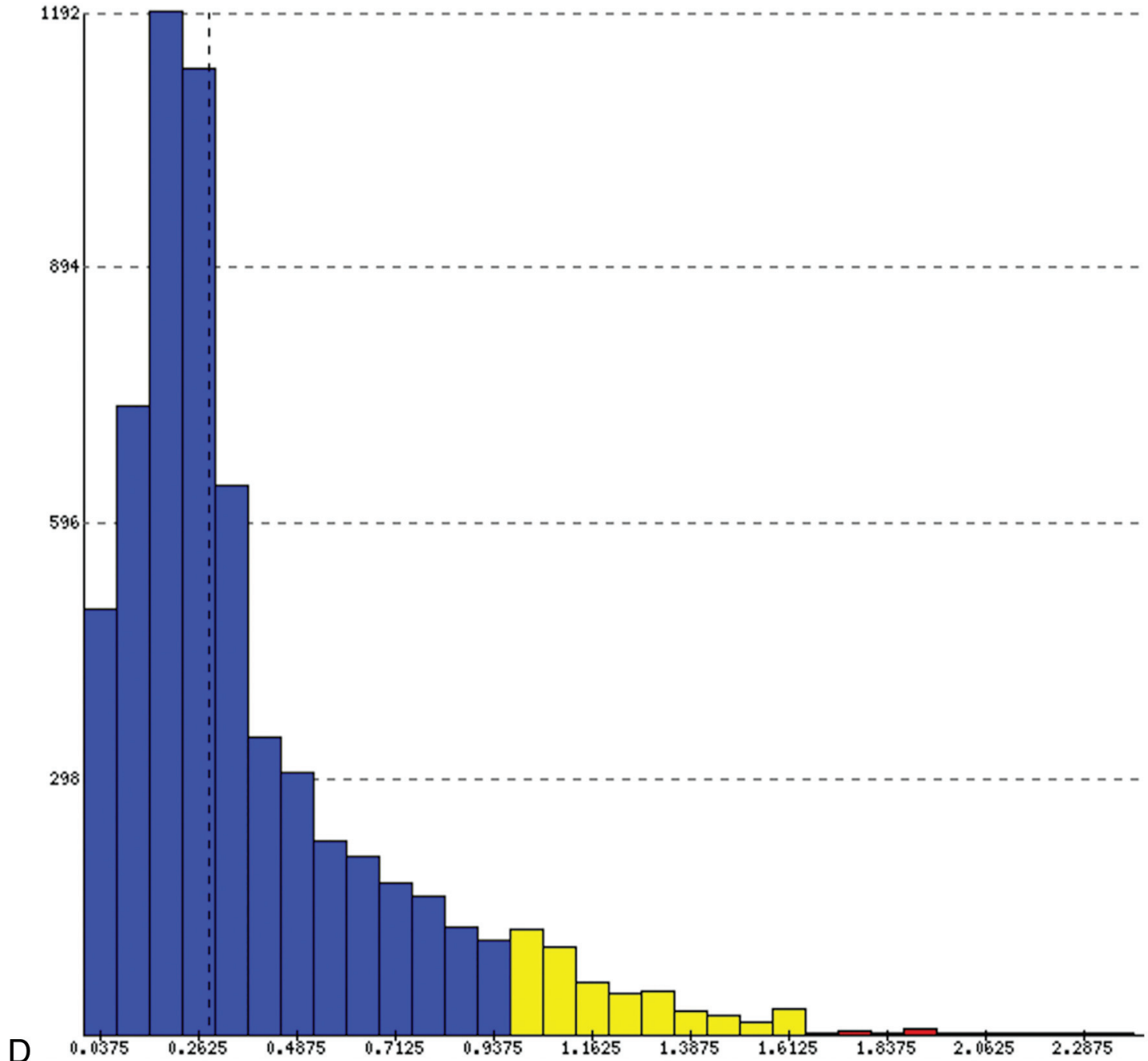

FIG 1. Representative examples of treatment effect $(A-D)$ and recurrent tumor $(E-H)$ in 2 patients with previously resected and irradiated glioblastomas. Contrast-enhancing lesions on postcontrast Tl-weighted $(A$ and $E$ ) and $\Delta T 7(B$ and $F)$ images. Output FTB maps superimposed on the contrast-enhanced Tl-weighted images $\left(C\right.$ and $G$ ). Blue represents areas of low blood volume ( $\mathrm{FB}_{\text {low }}$ ), and red represents areas of high blood volume $\left(\mathrm{FTB}_{\text {high }}\right)$. Histograms $\left(D\right.$ and $H$ ) show all voxels of the contrast-enhancing volume classified into the respective $\mathrm{FTB}_{\text {low, }} \mathrm{FTB}_{\text {mid }}(\mathrm{yellow})$, and $\mathrm{FTB}_{\text {high }}$ classes, which is based on the rCBV thresholds of 1.0 and 1.75 . 

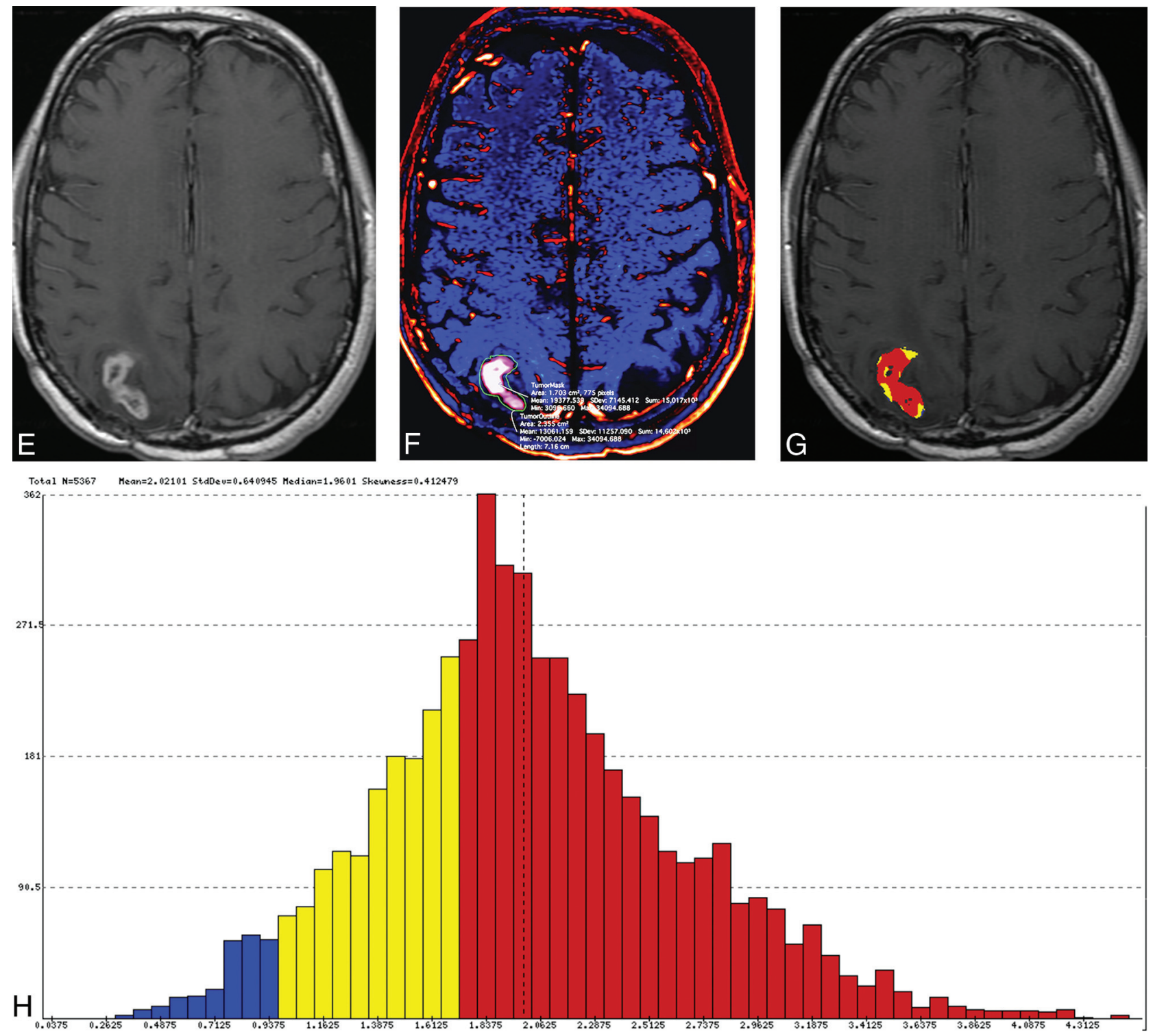

FIG 1. Continued.

rCBV of $\leq 1.0$; $\mathrm{FTB}_{\text {mid }}$, percentage of voxels with $\mathrm{rCBV}$ between 1.0 and 1.75; and $\mathrm{FTB}_{\text {high }}$, percentage of voxels with $\mathrm{rCBV}$ of $\geq 1.75$. Percentage values from the 3 FTB classes totaled $100 \%$. Mean rCBV values of the contrast-enhancing VOIs were generated for each patient. Volumetric images of the contrast-enhancing lesion superimposed on the FTB map containing colored voxels of each class $\left(\mathrm{FTB}_{\text {low }}=\right.$ blue; $\mathrm{FTB}_{\text {mid }}=$ yellow; $\mathrm{FTB}_{\text {high }}=$ red) and a histogram displaying voxels for the entire contrastenhancing VOI were also produced (Fig 1).

\section{Histopathologic Examination}

A team of 2 neuropathologists with 2 (J.L.) and 21 (D.E.B.) years of neuro-oncology-pathology experience was blinded to clinical and MR imaging results. Histopathologic examination of tissue specimens was performed by consensus agreement in a single session, with the reviewers providing only a single assessment for each sample. From each resection or biopsy, sections from different areas of the entire tissue submitted for histopathology were evaluated. To determine a single histopathologic diagnosis for a given case, the team evaluated whether each section consisted of TE (defined as the absence of neoplastic cells or the presence of scattered infiltrating atypical cells but without a focal group or a solid sheet of neoplastic cells) or tumor (defined as the presence of any group or a solid sheet of neoplastic cells with or without superimposed treatment-related changes). For the purpose of this study, scattered atypical cells were classified as TE because it was not absolutely certain to the neuropathologists whether the atypical cells represented radiation effect or treated-but-viable glioma cells. Second, the resolution of today's perfusion MR imaging is not high enough to detect scattered atypical cells that are otherwise only identified microscopically.

\section{Qualitative Analysis}

Five physician raters who are involved in the care of patients with glioma, consisting of 3 neuro-oncologists (R.T., S.N., L.R.) with varying levels of experience $(6,8$, and 35 years), a radiation 
Table 1: Patient demographics and clinicopathologic information ${ }^{\mathrm{a}}$

\begin{tabular}{|c|c|c|c|}
\hline & TE $(n=17)$ & $\begin{array}{l}\text { Tumor } \\
(n=30)\end{array}$ & Total $(n=47)$ \\
\hline \multicolumn{4}{|l|}{ Age (yr) } \\
\hline Mean (SD) & $56(10)$ & $55(13)$ & $55(12)$ \\
\hline Range & $38-77$ & $20-80$ & $20-80$ \\
\hline \multicolumn{4}{|l|}{ Sex } \\
\hline Male & $11(65 \%)$ & $18(60 \%)$ & $29(62 \%)$ \\
\hline Female & $6(35 \%)$ & $12(40 \%)$ & $18(38 \%)$ \\
\hline \multicolumn{4}{|l|}{$\begin{array}{l}\text { Interval time between end of } \\
\text { radiation therapy and } \\
\text { surgery (mo) }\end{array}$} \\
\hline Median (range) & $11.4(0.6-60.4)$ & $10.7(1.3-101.5)$ & $10.9(0.6-101.5)$ \\
\hline $\begin{array}{l}\text { Bevacizumab at time of } \\
\text { surgery }\end{array}$ & $2(12 \%)$ & $3(10 \%)$ & $5(11 \%)$ \\
\hline \multicolumn{4}{|l|}{ Surgical procedure } \\
\hline Biopsy & $3(18 \%)$ & $4(13 \%)$ & $7(15 \%)$ \\
\hline$>90 \%$ resection & $3(18 \%)$ & $9(30 \%)$ & $12(25 \%)$ \\
\hline Gross total resection & $11(64 \%)$ & $17(57 \%)$ & $28(60 \%)$ \\
\hline \multicolumn{4}{|l|}{ HGG histopathology } \\
\hline $\begin{array}{l}\text { Anaplastic astrocytoma, } \\
\text { WHO grade III }\end{array}$ & $2(12 \%)$ & 0 & $2(4 \%)$ \\
\hline $\begin{array}{l}\text { Glioblastoma, WHO grade } \\
\text { IV }\end{array}$ & 15 (88\%) & $29(97 \%)$ & $44(94 \%)$ \\
\hline $\begin{array}{l}\text { Gliosarcoma, WHO grade } \\
\text { IV }\end{array}$ & 0 & $1(3 \%)$ & $1(2 \%)$ \\
\hline \multicolumn{4}{|l|}{ HGG molecular features } \\
\hline IDH wild-type & $11(65 \%)$ & $11(37 \%)$ & $22(47 \%)$ \\
\hline IDH mutant & 0 & $2(7 \%)$ & $2(4 \%)$ \\
\hline Unknown IDH status & $6(35 \%)$ & $17(56 \%)$ & $23(49 \%)$ \\
\hline MGMT-unmethylated & $6(35 \%)$ & $15(50 \%)$ & $21(45 \%)$ \\
\hline MGMT-methylated & $6(35 \%)$ & $8(27 \%)$ & $14(30 \%)$ \\
\hline Unknown MGMT status & $5(30 \%)$ & $7(23 \%)$ & $12(25 \%)$ \\
\hline
\end{tabular}

Note:-IDH indicates isocitrate dehydrogenase; MGMT, O-6-methylguanine-DNA methyltransferase; WHO, World Health Organization.

${ }^{a}$ Percentage values in parentheses for sex, bevacizumab at time of the operation, surgical procedure, HGG histopathology, and HGG molecular features are percentages relative to the number of patients in each column.

${ }^{b}$ Patient received a dose of bevacizumab within 1 month of the surgical procedure for suspected recurrence.

oncologist (S.G.S.) with 14 years of experience, and a neuroradiologist (N.F.) with 25 years of experience, were blinded to clinical and histopathology information. Each rater was given a PowerPoint file (Microsoft, Redmond, Washington) consisting of representative MR images and numeric perfusion values of a patient's contrast-enhancing lesion (On-line Fig 2). The file consisted of 3 sample patients drawn from the total pool of 47 patients, one with histopathologically confirmed TE and the other 2 with histopathologically confirmed tumor. Anonymized data of the remaining 44 patients, who were presented in random order, followed the 3 sample patients. For all patients, 2 representative axial images of the segmented contrast-enhancing mass with 2 corresponding axial images of the color FTB map and the histogram and FTB percentages of the entire contrast-enhancing lesion were provided. Of note, although only 2 representative image slices of a patient's lesion were provided for visualization, the histogram data and percentage values, which represented the entire contrast-enhancing volume, were provided. Raters recorded whether they thought that the data represented TE or tumor and whether they would hypothetically change treatment on the basis of FTB. A change in treatment was defined as a change in surgical or medical management and excluded the option for shorter interval imaging surveillance. For qualitative image interpretation, we compared the consensus decision (representing most decisions among all raters) with the actual histopathologic diagnosis. For decisions of treatment change, we compared the consensus decision with the actual treatment decision that was made at the time of the patient's real-time care, which was largely based on histopathologic assessment.

\section{Statistical Analyses}

Descriptive statistics were used to report patient demographics and perfusion metrics. We used the nonparametric Mann-Whitney test to compare FTB classes and rCBV between the TE and tumor groups. The performance of each FTB class and rCBV to distinguish TE and tumor was evaluated with the area under the receiver operating characteristic curve. To determine whether the use of both $\mathrm{FTB}_{\text {low }}$ and $\mathrm{FTB}_{\text {high }}$ improved performance, we first used the Youden index to determine the percentage values that yielded the best sensitivity and specificity; then, we assigned a score of zero for cases that did not meet both conditions and a score of 1 for those that did. We subsequently used the Fisher exact test to assess significance between the groups and determined the sensitivity and specificity for this method. A $P<.05$ was considered statistically significant for all analyses.

For analysis of the qualitative data, we included only the 44 evaluated patients and excluded the 3 sample patients. The intraclass correlation coefficient (ICC) was used to assess agreement among the 5 raters for FTB interpretation and the decision to change treatment based on FTB. Agreement between the consensus scores for FTB interpretation and actual histopathologic diagnosis and between hypothetic and real-time treatment changes was also assessed with the ICC, using the following model of ICC interpretation: $<0.40$, poor; $0.40-0.75$, fair-togood; $\geq 0.75$, excellent. ${ }^{18}$

All statistical analyses were performed with $\mathrm{R}$ statistical and computing software (Version 3.4.0; http://www.r-project.org/); graphs were created with GraphPad Prism software for illustrative purposes (Version 8.0.1; GraphPad Software, San Diego, California).

\section{RESULTS}

\section{Patients}

Table 1 summarizes patient demographics and histopathologic and molecular tumor information. Seven patients underwent a biopsy, while 40 had a resection ( 12 had $>90 \%$ and 28 had gross total resection). Averages of 5 (range, 1-12) and 6 (range, 1-16) representative sections per the entire volume of submitted tissue specimen were analyzed in the biopsy and resection groups, respectively. On the basis of histopathologic examination, 17 were classified as TE (consisting of 4 samples with no tumor cells 
Table 2: Mean values of FTB classes and normalized rCBV in histopathologically defined treatment effect and tumor groups $^{\mathrm{a}}$

\begin{tabular}{lccc}
\hline & TE & Tumor & P Values \\
\hline FTB $_{\text {low }}$ & $54.8(22.3)$ & $33.1(20.8)$ & .002 \\
FTB $_{\text {mid }}$ & $27.0(15.4)$ & $21.3(11.3)$ & .16 \\
FTB $_{\text {high }}$ & $18.2(14.4)$ & $45.5(22.6)$ & $<.001$ \\
rCBV & $1.2(0.6)$ & $2.1(1.0)$ & $<.001$ \\
\hline
\end{tabular}

${ }^{a}$ Values are reported as mean (standard deviation), except for $P$ values.
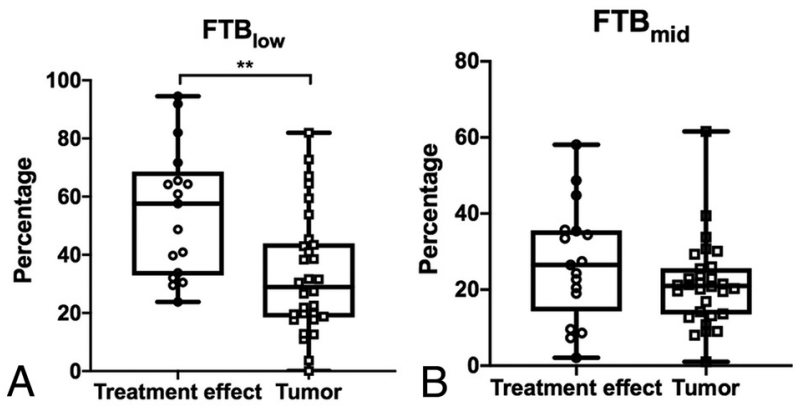

FTB high
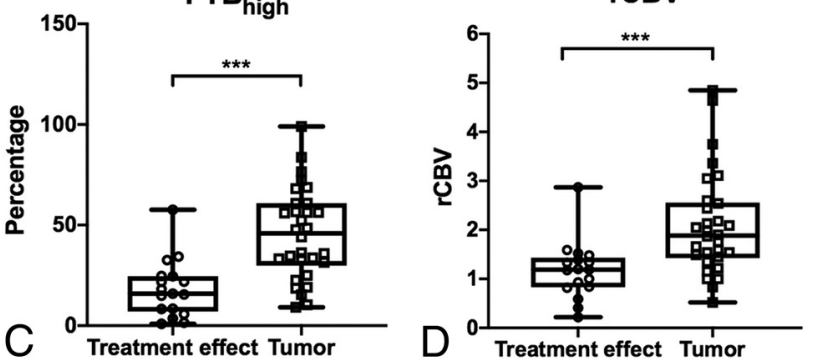

FIG 2. Boxplots of the relationship between FTB and normalized rCBV in 2 histopathologically defined groups: treatment effect and recurrent tumor. Open circles and squares represent individual measurements. The upper and lower limits of the whiskers represent the minimum and maximum of all of the data. Double asterisks indicate $P<.01$; triple asterisks, $P<.001$.

and 13 with scattered infiltrating atypical cells), and 30, as recurrent tumor.

\section{Quantitative FTB and rCBV}

Differences in mean $\mathrm{FTB}_{\text {low }}$, $\mathrm{FTB}_{\text {high }}$ and rCBV of the contrastenhancing lesion volume were significant between TE and tumor $(P=.002,<.001$, and $<.001$, respectively), with tumor having higher $\mathrm{FTB}_{\text {high }}$ and $\mathrm{rCBV}$ and lower $\mathrm{FTB}_{\text {low }}$ than TE (Table 2 and Fig 2). No significance was found with $\operatorname{FTB}_{\text {mid }}(P=.16)$.

Areas under the receiver operating characteristic curve for using FTB and rCBV to distinguish TE and tumor were the following: 0.77 for $\mathrm{FTB}_{\text {low }}$ (95\% CI, 0.64-0.90; $P=.002$ ), 0.63 for $\mathrm{FTB}_{\text {mid }}(95 \% \mathrm{CI}, 0.44-0.81 ; P=.16), 0.85$ for $\mathrm{FTB}_{\text {high }}(95 \% \mathrm{CI}$, $0.74-0.97 ; P<.001$ ), and 0.81 for $\operatorname{rCBV}$ (95\% CI, 0.69-0.94; $P<.001$ ) (Fig 3). The $\mathrm{FTB}_{\text {high }}$ cut-point of $>24.9 \%$ yielded a sensitivity of $80 \%$ and a specificity of $82 \%$, and the $\mathrm{FTB}_{\text {low }}$ cut-point of $<28.5 \%$ yielded a sensitivity of $50 \%$ and specificity of $94 \%$ for tumor prediction. The use of both cut-points showed significance in differentiating tumor from TE $(P<.001)$, with a sensitivity of $100 \%$ and specificity of $47 \%$. The optimal rCBV cut-point for

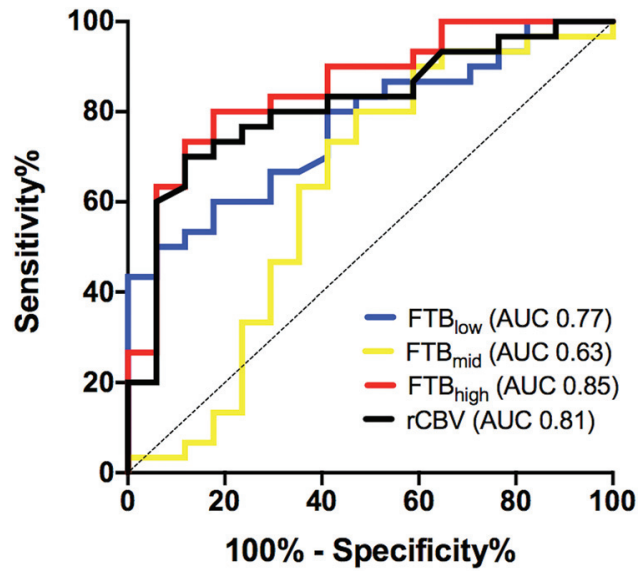

FIG 3. Receiver operating characteristic curves for the use of fractional tumor burden classes and normalized rCBV to differentiate tumor from treatment effect.

identifying tumor was found to be $>1.53$, yielding a sensitivity of $70 \%$ and specificity of $88 \%$.

\section{Qualitative Analysis}

Agreement among the 5 physicians for the use of FTB to differentiate TE from tumor was fair-to-good (ICC $=0.48$ ). When we compared the consensus decision with the actual histopathology, agreement improved $(\mathrm{ICC}=0.70)$. Of the total number of cases with histopathologically confirmed tumor $(n=28)$ and TE $(n=16)$, there were disagreements in $7 \%(2 / 28)$ of tumor and $25 \%(4 / 16)$ of TE cases (Fig $4 A$ ).

Agreement among the raters when asked whether they would hypothetically change treatment on the basis of their interpretation of FTB was fair-to-good (ICC $=0.48$ ). The consensus decision was to hypothetically change treatment in $93 \%$ $(26 / 28)$ of tumor cases and not to change treatment in $75 \%$ $(12 / 16)$ of TE cases. When we compared the consensus decision with the actual treatment plan, agreement was fair-togood (ICC $=0.46)$. Of the histopathologically confirmed tumor and TE groups, there were disagreements in $7 \%(2 / 28)$ of tumor and $44 \%(7 / 16)$ of TE cases (Fig $4 B$ ). In this latter TE group, all disagreements occurred in cases in which the surgical specimen showed scattered atypical cells and none in which the specimen showed no tumor cells (Fig 4C).

\section{DISCUSSION}

Our results show that the use of $2 \mathrm{rCBV}$ thresholds to define low and high fractional tumor burden of the contrast-enhancing volume allows differentiation of tumor and TE in the recurrent glioblastoma setting. Of the $3 \mathrm{FTB}$ classes and $\mathrm{rCBV}$ parameters, $\mathrm{FTB}_{\text {high }}$ performed the best for this task. In addition, we found good consensus agreement among 5 physicians for the use of FTB to differentiate TE from tumor and to inform potential treatment-related decision-making.

Given the variability of previously published mean rCBV thresholds to differentiate TE from tumor (with a reported range of 0.9-2.15 based on a recent meta-analysis), ${ }^{6}$ we selected predefined values on the low and high ends of the range to define 

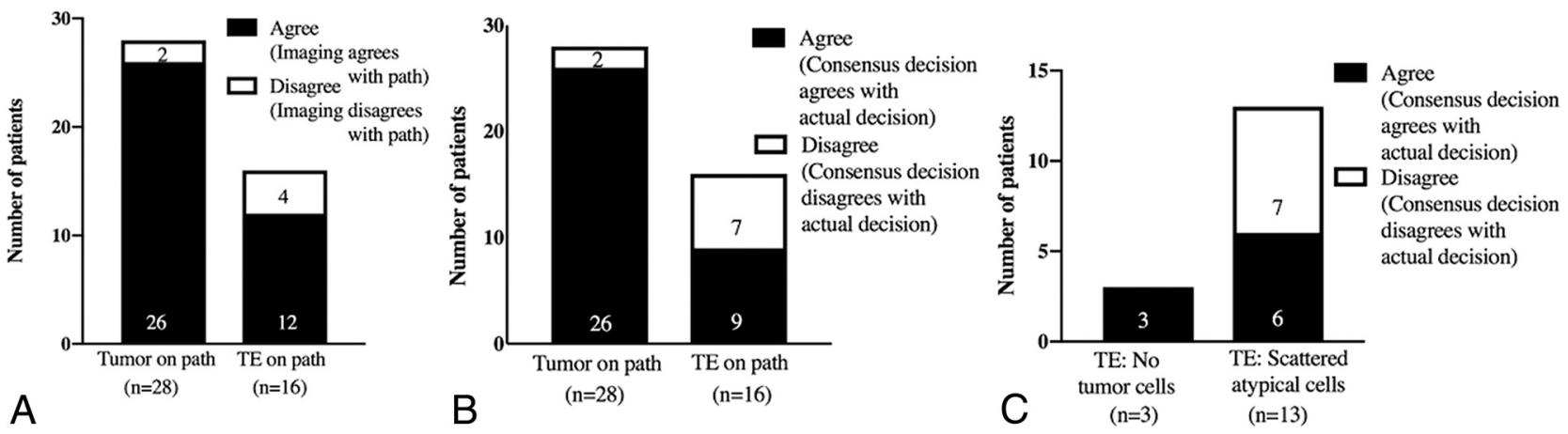

FIG 4. Agreement between the consensus (among 5 physician raters) qualitative interpretation of imaging and the actual histopathologic diagnosis $(A)$. Agreement between the hypothetic consensus decision to change treatment plans and the actual (real-time) management plans ( $B$ and $C)$.

fractions of the contrast-enhancing volume with low and high blood volume, respectively. On the basis of prior radiology-pathology correlative studies, areas of TE tend to have low blood volume and areas of tumor tend to have high blood volume on perfusion MR imaging. ${ }^{2,3,19}$ We did not generate the optimal thresholds to use from our own dataset (to minimize institutional bias in generating the values) and opted to use values that have been tested or validated in prior studies: On the low end of the rCBV range, a threshold of 1.0 has been used to reliably distinguish TE and tumor, ${ }^{2,3}$ and on the high end, a threshold of 1.75 has been used to identify aggressive tumors. ${ }^{8,10}$ In our study, the use of 2 thresholds allowed the delineation of 3 FTB classes; we found that TE had significantly higher $\mathrm{FTB}_{\text {low }}$ than tumor and that tumor had significantly higher $\mathrm{FTB}_{\text {high }}$ than TE. In comparing these metrics, $\mathrm{FTB}_{\text {high }}$ performed better than $\mathrm{FTB}_{\text {low }}$ for tumor identification. The use of both $\mathrm{FTB}_{\text {high }}$ and $\mathrm{FTB}_{\text {low }}$ percentage cut-points improved the sensitivity of tumor diagnosis to $100 \%$, but specificity remained low. We postulate that $\mathrm{FTB}_{\text {high }}$ is a more robust marker than $\mathrm{FTB}_{\text {low }}$ due to tumoral heterogeneity, in which previously treated tumors can have regional and interspersed areas of both high and low blood volume, presumably related to varying degrees of angiogenesis and necrosis, respectively. ${ }^{20}$ In contrast, areas of pure TE and radiation necrosis tend to consistently show low blood volume. ${ }^{21}$ Sampling error, which is further discussed in the limitations, may also help to explain the lower specificity found in this study. FTB $_{\text {mid, }}$ which includes all $\mathrm{rCBV}$ values between 1.0 and 1.75 , did not reliably differentiate TE from tumor, likely because of overlapping values in this range found in samples with both TE and tumor, which is consistent with findings in the study of Barajas et al. ${ }^{20}$ Nonetheless, a strength of using FTB is that it is less dependent on the magnitude of rCBV values, except to classify voxels within a FTB class, and it has been shown to better approximate tumor volume fraction. $^{2}$

We did not correlate perfusion results with prognostic clinical end points such as overall survival because there were insufficient patient numbers to perform a meaningful analysis. Rather, we assessed whether FTB data could be used to predict the histopathologic diagnosis and inform short-term management plans. Agreement between the consensus assessment of disease based on FTB and the actual histopathologic diagnosis was good. In keeping with the imaging interpretation, the consensus decision was to hypothetically change treatment in $93 \%$ (26/28) of tumor cases and not to change treatment in $75 \%$ $(12 / 16)$ of TE cases. This is in the context of all patients undergoing surgical intervention in real-time because of concerns for tumor progression at the time of the clinical MR imaging and suggests the potential role of this approach to help triage patients who may or may not need an operation for diagnosis. Most of the disagreement in treatment changes occurred in the histopathologically defined TE group; upon further analysis, all disagreements in this group occurred in cases in which histopathology showed scattered atypical cells. This may be because, in real-time, the decision to change treatment considered the uncertainty of whether the atypical cells represented radiation-related change or treated-but-viable glioma cells and other clinical factors (such as patient age, Karnofsky Performance Status Scale, tumor molecular status, and patient desires) that were not incorporated in our study. Nonetheless, our findings are in keeping with those of Geer et al, ${ }^{22}$ who showed that the addition of DSC and arterial spin-labeling perfusion imaging impacted and changed management plans in 8.5\% (5/59) of patient care episodes and significantly increased physician confidence in treatment plans. In contrast to that study, which included only qualitative image interpretation, we show the potential of a combined approach using quantitative and qualitative data to inform treatment-related decisionmaking.

Limitations of this study should be considered. First, the retrospective nature of this study consisting of a single institutional dataset and small sample size limits the generalizability of our results. Second, acquisition of DSC-MR imaging during the study period was performed without preload dosing, which can confound rCBV estimates. ${ }^{7,23}$ Of note, $\mathrm{Hu}$ et $\mathrm{al}^{5}$ showed that the use of IB Neuro software, which was used in this study, generated rCBV metrics, including FTB, that were highly correlated even between nonpreload- and preload-dose-corrected conditions. Schmainda et $\mathrm{al}^{15}$ also recently showed that DSC imaging using a low flip angle $\left(30^{\circ}\right)$ and no preload dose produces $\mathrm{rCBV}$ values similar to those of the conventional method of using an intermediate flip angle $\left(60^{\circ}\right)$ with preload and postprocessing leakage correction. Third, misregistration of VOIs 
due to geometric distortion in DSC is another potential source of error. Finally and most important, we used histopathologic examination of available tissue specimens from both surgical resection and, to a smaller extent, biopsies for ground truth diagnoses. Although we evaluated different representative sections of the entire submitted samples to determine a diagnosis, we could not provide histopathologic correlation for every voxel of the contrast-enhancing volume available on FTB. Because glioblastomas are markedly heterogeneous tumors, histopathologic diagnosis of samples from separate locations may differ; even with a given sample, diagnostic agreement among pathologists can vary. ${ }^{24}$ However, despite the real possibility of sampling error, histopathologic assessment remains the "criterion standard" for determining disease status and often influences treatment-related decision-making. Taking these issues into consideration, we show the potential of FTB to distinguish TE from tumor, and we have yet to fully explore its role as an alternative means to provide accurate diagnostic and prognostic information, particularly given the limitations of histopathologic evaluation.

\section{CONCLUSIONS}

The use of $2 \mathrm{rCBV}$ thresholds (1.0 and 1.75) to define $\mathrm{FTB}_{\text {low }}$ and $\mathrm{FTB}_{\text {high }}$ provided good spatial visualization and quantification of contrast-enhancing volume fraction with low and high blood volume, respectively, and they performed well to differentiate tumor from TE in the recurrent glioblastoma setting. In addition, combining qualitative image interpretation with quantitative FTB data can help inform clinical decisionmaking, with such decisions closely mirroring actual treatment decisions made for groups with histopathologically confirmed tumor and no tumor cells. Larger prospective studies, however, are needed to validate this method for use in real-time clinical decision-making and for correlation with important clinical outcomes.

Disclosures: Scott G. Soltys—UNRELATED: Consultancy: Inovio Pharmaceuticals.

\section{REFERENCES}

1. Wen PY, Macdonald DR, Reardon DA, et al. Updated response assessment criteria for high-grade gliomas: Response Assessment in Neuro-Oncology Working Group. J Clin Oncol 2010;28:1963-72 CrossRef Medline

2. Hu LS, Eschbacher JM, Heiserman JE, et al. Reevaluating the imaging definition of tumor progression: perfusion MRI quantifies recurrent glioblastoma tumor fraction, pseudoprogression, and radiation necrosis to predict survival. Neuro Oncol 2012;14:919-30 CrossRef Medline

3. Prah MA, Al-Gizawiy MM, Mueller WM, et al. Spatial discrimination of glioblastoma and treatment effect with histologicallyvalidated perfusion and diffusion magnetic resonance imaging metrics. J Neurooncol 2018;136:13-21 CrossRef Medline

4. Jain R, Scarpace L, Ellika S, et al. First-pass perfusion computed tomography: initial experience in differentiating recurrent brain tumors from radiation effects and radiation necrosis. Neurosurgery 2007;61:778-86; discussion 786-87 Medline

5. Hu LS, Kelm Z, Korfiatis P, et al. Impact of software modeling on the accuracy of perfusion MRI in glioma. AJNR Am J Neuroradiol 2015;36:2242-49 CrossRef Medline
6. Patel P, Baradaran H, Delgado D, et al. MR perfusion-weighted imaging in the evaluation of high-grade gliomas after treatment: a systematic review and meta-analysis. Neuro Oncol 2017;19:118-27 CrossRef Medline

7. Paulson ES, Schmainda KM. Comparison of dynamic susceptibilityweighted contrast-enhanced MR methods: recommendations for measuring relative cerebral blood volume in brain tumors. Radiology 2008;249:601-13 CrossRef Medline

8. Law M, Young RJ, Babb JS, et al. Gliomas: predicting time to progression or survival with cerebral blood volume measurements at dynamic susceptibility-weighted contrast-enhanced perfusion MR imaging. Radiology 2008;247:490-98 CrossRef Medline

9. Prager AJ, Martinez N, Beal K, et al. Diffusion and perfusion MRI to differentiate treatment-related changes including pseudoprogression from recurrent tumors in high-grade gliomas with histopathologic evidence. AJNR Am J Neuroradiol 2015;36:877-85 CrossRef Medline

10. Gasparetto EL, Pawlak MA, Patel SH, et al. Posttreatment recurrence of malignant brain neoplasm: accuracy of relative cerebral blood volume fraction in discriminating low from high malignant histologic volume fraction. Radiology 2009;250:887-96 CrossRef Medline

11. Cha S, Tihan T, Crawford F, et al. Differentiation of low-grade oligodendrogliomas from low-grade astrocytomas by using quantitative blood-volume measurements derived from dynamic susceptibility contrast-enhanced MR imaging. AJNR Am J Neuroradiol 2005;26:266-73 Medline

12. Boxerman JL, Zhang Z, Safriel Y, et al. Early post-bevacizumab progression on contrast-enhanced MRI as a prognostic marker for overall survival in recurrent glioblastoma: results from the ACRIN 6677/RTOG 0625 Central Reader Study. Neuro Oncol 2013;15:94554 CrossRef Medline

13. Prah MA, Stufflebeam SM, Paulson ES, et al. Repeatability of standardized and normalized relative $\mathrm{CBV}$ in patients with newly diagnosed glioblastoma. AJNR Am J Neuroradiol 2015;36:1654-61 CrossRef Medline

14. Schmainda KM, Prah MA, Rand SD, et al. Multisite concordance of DSC-MRI analysis for brain tumors: results of a National Cancer Institute quantitative imaging network collaborative project. AJNR Am J Neuroradiol 2018;39:1008-16 CrossRef Medline

15. Schmainda KM, Prah MA, Hu LS, et al. Moving toward a consensus DSC-MRI protocol: validation of a low-flip angle single-dose option as a reference standard for brain tumors. AJNR Am J Neuroradiol 2019;40:626-33 CrossRef Medline

16. Bedekar D, Schmainda KM, Rand S, et al. Delta T1 (dT1) method as a tool to evaluate tumor progression in patients with brain cancer. J Clin Oncol 2011;29:e21056-56

17. Schmainda KM, Prah MA, Zhang Z, et al. Quantitative delta T1 (dT1) as a replacement for adjudicated central reader analysis of contrast-enhancing tumor burden: a subanalysis of the American College of Radiology Imaging Network 6677/Radiation Therapy Oncology Group 0625 Multicenter Brain Tumor Trial. AJNR Am J Neuroradiol 2019;40:1132-39 CrossRef Medline

18. Rosner B. Fundamentals of Biostatistics. Belmont: Thomson Brooks/ Cole; 2006

19. Hu LS, Baxter LC, Smith KA, et al. Relative cerebral blood volume values to differentiate high-grade glioma recurrence from posttreatment radiation effect: direct correlation between imageguided tissue histopathology and localized dynamic susceptibility-weighted contrast-enhanced perfusion MR imaging measurements. AJNR Am J Neuroradiol 2009;30:552-58 CrossRef Medline

20. Barajas RF Jr, Chang JS, Segal MR, et al. Differentiation of recurrent glioblastoma multiforme from radiation necrosis after external beam radiation therapy with dynamic susceptibility-weighted contrast-enhanced perfusion MR imaging. Radiology 2009;253:486-96 CrossRef Medline 
21. Li Z, Zhou Q, Li Y, et al. Mean cerebral blood volume is an effective diagnostic index of recurrent and radiation injury in glioma patients: a meta-analysis of diagnostic test. Oncotarget 2017;8:1564250 CrossRef Medline

22. Geer CP, Simonds J, Anvery A, et al. Does MR perfusion imaging impact management decisions for patients with brain tumors? A prospective study. AJNR Am J Neuroradiol 2012;33:556-62 CrossRef Medline
23. Hu LS, Baxter LC, Pinnaduwage DS, et al. Optimized preload leakage-correction methods to improve the diagnostic accuracy of dynamic susceptibility-weighted contrast-enhanced perfusion MR imaging in posttreatment gliomas. AJNR Am J Neuroradiol 2010;31:40-48 CrossRef Medline

24. Holdhoff M, Ye X, Piotrowski AF, et al. The consistency of neuropathological diagnoses in patients undergoing surgery for suspected recurrence of glioblastoma. J Neurooncol 2019;141:347-54 CrossRef Medline 